\title{
Universidad y pandemia: la comunicación en la educación a distancia
}

\author{
University and pandemic: communication in distance education
}

\author{
Dra. María Jesús Fernández Torres \\ Universidad de Málaga | Calle de León Tolstoi, s/n, 29010, Málaga | España | \\ https://orcid.org/0000-0002-8776-493X | mariajesusfernandez@uma.es \\ Dra. Rocío Chamizo Sánchez \\ Universidad de Málaga | Calle de León Tolstoi, s/n, 29010, Málaga | España | \\ https://orcid.org/0000-0003-4426-8298 | rcs@uma.es

\section{Rocío Sánchez Villarrubia} \\ Junta de Extremadura | Ctra. Rosalejo, 1, 10300 Navalmoral de la Mata, Cáceres | España | \\ https://orcid.org/0000-0001-9158-1530 | rsanchezv23@educarex.es
}

Fechas | Recepción: 27/12/2020 | Aceptación: 27/02/2021

\section{Resumen}

El estado de alarma causado por la COVID-19 invadió precipitadamente nuestras vidas con implicaciones no solo políticas, económicas o sanitarias, sino también educativas. La educación tuvo que adaptarse a un nuevo escenario en donde las Tecnologías de la Información y la Comunicación asumieron el protagonismo absoluto. Profesorado y alumnado tuvieron que acomodarse, de forma improvisada, a una educación a distancia para la que no estaban preparados, con unas materias cuyos programas estaban diseñados para una educación presencial y no exclusivamente virtual. Esta investigación pretende conocer la adaptación de la universidad española a este nuevo escenario, prestando especial atención a la comunicación desarrollada entre el docente y el discente, como pieza clave de una educación efectiva (Aliste, 2007). Se propone una investigación exploratoria con un cuestionario estructurado como herramienta metodológica dirigido a la comunidad educativa universitaria española. Los resultados muestran dificultad por parte

\begin{abstract}
The alarm state caused by COVID-19 hastily invaded our lives with not only political, economic or sanitary implications but with educational ones too. Education had to adapt itself to a new scenario where the ICTs assumed the leading role. Faculty and students had to adapt to distance education in an improvised way which neither of them were ready for, with subjects which were designed for a face-to-face education and not for an exclusively virtual education. This investigation intends to know the adaption of the Spanish universities to this new scenario, paying special attention to the communication between the faculty and students as the key for an effective education (Aliste 2007). An exploratory research with a structured survey is proposed as the methodological tool that is aimed to the Spanish educational campus community. Results show difficulties on the part of professors and students when facing the virtual learning and, at the same time, this results warn of the needs of the online education which are pre-sent in teaching plans to guarantee the
\end{abstract}


de profesores y estudiantes a la hora de enfrentarse a la enseñanza telemática y advierten de la necesidad de una educación en línea que esté soportada en planificados programas docentes que garanticen la efectividad del proceso de enseñanzaaprendizaje. Tanto profesorado como alumnado señalan el empobrecimiento de la comunicación didáctica y social en la educación en línea desarrollada durante el estado de alarma. Las herramientas más empleadas y mejor valoradas por parte de los agentes del proceso han sido la videoconferencia y el correo electrónico.

Palabras clave: educomunicación, enseñanza superior, comunicación interpersonal, innovación educacional, Covid-19. effectiveness of the teaching-learning process. Faculty and learners point out the impoverishment of the didactic and social communication in online education developed during the state of alarm. The most used and best valued tools are the videoconference and the email.

Keywords: educommunication, higher education, interpersonal communication, educational innovation, Covid-19.

\section{INTRODUCCIÓN}

El estado de alarma causado por la COVID-19 provocó el cierre de los centros educativos a nivel mundial. La educación tuvo que adaptarse rápidamente a un nuevo escenario en el que las Nuevas Tecnologías de la Comunicación y de la Información asumieron el protagonismo. Profesores y alumnos tuvieron que acomodarse, de manera improvisada, a una educación a distancia carente del diseño y de la planificación necesaria que pudiese garantizar la calidad del servicio educativo y el cumplimiento satisfactorio de los objetivos académicos.

Asistimos a una educación online de emergencia en respuesta a una situación de crisis sanitaria que ha dado lugar al traspaso, temporal, de la enseñanza en el aula tradicional al e-learning. No obstante, tal y como señalan Hodges et al. (2020), esta adaptación de lo presencial a lo virtual no responde a unos preceptos de calidad y, por consiguiente, no deberíamos equiparlo a la educación en línea al uso, dado que este tipo de educación requiere de un tiempo de preparación del que se ha carecido.

La propuesta que planteamos con este estudio es conocer cómo se han adaptado las universidades españolas al nuevo escenario. El artículo parte del planteamiento de unos objetivos de investigación y de unas preguntas de análisis que marcarán el desarrollo del estudio. A continuación, se presenta la herramienta metodológica utilizada para alcanzar los resultados expuestos e inferir las conclusiones finales de la investigación, realizando la pertinente discusión fundamentada en el estado del arte realizado en la revisión teórica y en el planteamiento del problema. Los centros contemplados en la investigación responden a la modalidad de enseñanza presencial, con una incorporación, cada vez mayor, de los entornos virtuales en los procesos de enseñanza-aprendizaje en respuesta a los planteamientos derivados del Marco Europeo de Educación Superior, al desarrollo creciente de las Nuevas Tecnologías y a las demandas de un alumnado digitalmente conectado.

Lejos de poder realizar una comparación real y objetiva entre la enseñanza presencial y la telemática en la educación superior, dado que la educación en línea que ha tenido lugar no ha sido fruto de un propósito educativo contemplado en el diseño de elaborados proyectos 
docentes, vamos a poder valorar el estado en el que se encuentran las universidades en modalidad presencial en materia de capacitación digital del profesorado, en la disponibilidad, empleo y utilidad de los recursos y herramientas digitales, así como la disposición, hacia este tipo de enseñanza-aprendizaje, del profesorado y del alumnado. Por otra parte, considerando que en este salto al entorno virtual se ha visto especialmente afectada la comunicación entre el docente y el estudiantado, el propósito de esta investigación será valorar los resultados obtenidos bajo el enfoque de la comunicación desarrollada como elemento clave de una práctica educativa efectiva.

Poniendo el foco en el estado de la cuestión sobre el tema que se aborda, y en el que se profundizará a continuación, cabe destacar la escasez de bibliografía relacionada con la comunicación profesor-alumno en entornos virtuales. Autores como Mahle (2011) o Joo et al. (2011) destacan la importancia de una adecuada interacción para favorecer la motivación y el entusiasmo del alumnado, en un proceso de aprendizaje que se vuelve más autónomo para el estudiante y en el que el docente adquiere, necesariamente, el rol de orientador (Vilanova, 2016). En este nuevo escenario, en el que lo digital adquiere protagonismo y las relaciones personales se distancian, la tutoría virtual se convierte en una herramienta de comunicación esencial (Hernández-Sánchez et al., 2015). El estudio presentado pretende, por consiguiente, arrojar información y contribuir a la mejora de la educación universitaria y al fortalecimiento de la relación profesor-alumno, a través del uso planificado de las herramientas de comunicación disponibles.

\subsection{Objetivos y metodología}

El objetivo principal de esta investigación es conocer y valorar la comunicación desarrollada entre el profesorado y el alumnado a partir de la adaptación a la enseñanza virtual experimentada por las universidades españolas en modalidad presencial, a consecuencia del estado de emergencia experimentado por la crisis sanitaria mundial a causa del coronavirus. Por su parte, los objetivos secundarios que complementan el estudio son: conocer y valorar el estado de adaptación al entorno virtual en el que se encuentran las universidades en España; identificar las ventajas y/o inconvenientes que ha supuesto dicha adaptación en la comunicación entre docente y discente, así como determinar las herramientas y recursos de comunicación de mayor y menor valoración por parte de estudiantes y docentes en la enseñanza a distancia.

Los objetivos planteados pretenden dar respuestas a las siguientes cuestiones de análisis:

P1.- ¿qué comunicación se ha desarrollado entre los principales actores de la enseñanza universitaria (docente-discente) en el nuevo escenario sobrevenido por la COVID-19?

P2.- ¿qué grado de dificultad se ha encontrado en el proceso de enseñanza-aprendizaje, en el entorno virtual, por parte de profesorado y alumnado?

P3.- ¿qué herramientas se han empleado y cuáles han funcionado mejor a la hora de establecer la interacción profesor-alumno?

P4.- ¿qué valoración realizan dichos agentes de esta experiencia?

La investigación que aquí se aborda es descriptiva y de carácter exploratorio. Se ha sustentado en un intenso vaciado bibliográfico para construir el marco teórico, completándose con una metodología cuantitativa, centrándose en la encuesta como herramienta metodológica. 
La encuesta se lanzó para conocer cómo las universidades españolas que respondían a modalidad presencial antes del confinamiento provocado por la COVID-19 se han adaptado al nuevo escenario; conocer la capacitación de los docentes universitarios, así como la disponibilidad, empleo y utilidad de los recursos y herramientas digitales hacia este tipo de enseñanza, tanto por parte del profesorado como del estudiantado universitario, han sido también objeto de esta herramienta metodológica. Otro de sus objetivos era conocer la gestión de la comunicación llevada a cabo entre los públicos más importantes de la Educación Superior, es decir, alumnos y profesores. El universo, en definitiva, lo componen todo aquel profesorado o estudiantado miembro de cualquiera de los centros universitarios españoles, públicos o privados, con docencia presencial.

La encuesta la componían un total de 24 preguntas, 12 para el docente y 12 para el alumnado, además de las cuestiones discriminatorias iniciales referidas a variables demográficas (edad, género, comunidad...), titularidad de la universidad, así como rama de conocimiento y nivel de estudios cursados. Las preguntas a docentes y discentes, si bien versaban sobre aspectos coincidentes para ambos públicos, fueron diferentes en el planteamiento y en las opciones de respuesta, adaptándose en función de la casuística de cada uno de los roles. Dicho cuestionario se diseñó pretendiendo averiguar el conocimiento de las plataformas y recursos que las universidades habían utilizado durante la pandemia, el uso de las mismas por estudiantes y profesores antes del confinamiento, nivel de alfabetización digital, herramientas más utilizadas durante la pandemia para comunicarse entre profesores y alumnos, herramientas que los protagonistas consideraban que habían funcionado mejor en el proceso de enseñanzaaprendizaje así como en la comunicación para el fomento de relaciones personales, inconvenientes y ventajas de la enseñanza virtual respecto a la presencial, influencia de la enseñanza telemática en la mejora o en el empeoramiento de vínculo docente-alumno, experiencia comunicativa y grado de dificultad encontrado con la enseñanza online.

La encuesta fue contestada por un total de 711 personas, siendo 210 las respuestas facilitadas por docentes y 501 las contestadas por estudiantes de diferentes universidades. Dicha encuesta tuvo un margen de error +-3 y un nivel de confianza del $96 \%$.

Permaneció activa durante dos meses. De tal forma, se lanzó el 29 de abril de 2020 y se cerró el 29 de junio del mismo año. La muestra fue aleatoria y abarcó todo el país. La forma de proceder a la selección de la muestra y a la difusión del cuestionario fue la siguiente: en un primer momento, se realizó una búsqueda al azar de profesorado en el total de directorios ofrecidos por las universidades públicas y privadas españolas con modalidad presencial, estableciéndose un listado de contactos aleatorio a los que se les envió un correo electrónico explicándoles los objetivos del estudio e invitándoles a colaborar en el mismo, cumplimentando la encuesta y distribuyéndola entre su alumnado. Se contemplaron para el mailing todas las universidades españolas en modalidad presencial. Igualmente, fue difundida a través de las plataformas virtuales, de las redes sociales de las investigadoras (Facebook y Twitter) y de WhatsApp.

En la encuesta participaron, finalmente, profesorado y alumnado de las siguientes 37 universidades españolas, a saber: Universidad de Almería, Universidad Antonio Nebrija, Universidad Autónoma de Barcelona, Universidad Autónoma de Madrid, Universidad de Alcalá, Universidad de Alicante, Universidad de Cádiz, Universidad de Cantabria, Universidad de Castilla La Mancha, Universidad Complutense de Madrid, Universidad de Córdoba, Universidad de 
Extremadura, Universidad de Granada, Universidad de Huelva, Universidad de las Islas Baleares, Universidad de Jaén, Universidad de La Laguna, Universidad de La Rioja, Universidad de León, Universidad de Málaga, Universidad de Murcia, Universidad de Navarra, Universitat Oberta de Catalunya, Universidad de Oviedo, Universidad del País Vasco, Universidad Politécnica de Catalunya, Universidad Politécnica de Madrid, Universidad Ramón Llull, Universidad Rovira i Virgili, Universidad San Pablo C.E.U., Universidad de Santiago de Compostela, Universidad de Salamanca, Universidad de Sevilla, Universidad de Valencia, Universidad de Valladolid, Universidad de Vigo y Universidad de Zaragoza.

\section{REVISIÓN TEÓRICA Y PLANTEAMIENTO DEL PROBLEMA}

La enseñanza superior en Europa ha venido experimentando una importante transformación motivada por los cambios derivados del proceso de Bolonia y por el extraordinario desarrollo de las Tecnologías de la Información y las Comunicaciones (en adelante, TIC). El Espacio Europeo de Educación Superior ha supuesto la transición de un modelo educativo basado en metodologías de enseñanza pasivas a metodologías activas, en donde adquiere una especial importancia el proceso de aprendizaje y el desarrollo de habilidades en el estudiantado (Moreira et al., 2017; Raya y Gómez, 2018). En este nuevo escenario de la Educación Superior, las nuevas tecnologías no sólo se presentan como un instrumento o medio de información y comunicación, sino como un nuevo espacio social para la educación (Echeverría, 2002). De tal forma, los escenarios virtuales de enseñanza responden a las nuevas propuestas metodológicas requeridas por los cambios sociales, comunicacionales, tecnológicos y de educación a los que estamos asistiendo (Lorenzo et al., 2019).

Como señalan Chamizo y Fernández (2013), las Nuevas Tecnologías han irrumpido en el aula y las TIC aumentan la oferta educativa haciendo posibles nuevos modelos de enseñanza, desde la presencial a la distancia o a propuestas mixtas que incorporan las actividades en la clase y en el ciberespacio. Según las autoras, estas herramientas generan nuevas posibilidades para la interacción didáctica.

Durán (2016) destaca el gran potencial de la educación virtual en la Universidad como medio para mejorar las competencias genéricas y los aprendizajes de los estudiantes. Concluye que, para mejorar la calidad y la credibilidad de este tipo de enseñanza, en muchas ocasiones cuestionada, es preciso evaluar permanentemente la planificación de los procesos educativos, basada en el número de estudiantes atendidos, la disponibilidad del material instruccional, la calidad de los profesores, la calidad y pertinencia de las estrategias didácticas y la interacción con el alumno. En este sentido, Forsyth et al. (2010) identifican la estructura organizacional y la cultura como factores críticos de éxito para la calidad en la educación a distancia. Por otra parte, Shailendra et al. (2018) advierten de la necesidad de mejorar la calidad percibida en la educación online y apuestan por combinar las virtudes de la educación presencial y la telemática con el fin de perpetuar un equilibrio óptimo entre la tecnología y la educación de contacto.

La creación de espacios virtuales en la educación precisa que, para una adecuada implementación en el sistema, el docente dedique una cantidad de tiempo considerable a la preparación y al seguimiento de la actividad en el entorno virtual, creando contenidos específicos y fomentando la participación del estudiantado, proporcionándole retroalimentación inmediata a sus participaciones (Esquivel \& Canto, 2018). Igualmente, requiere que el profesorado esté capacitado en el uso de las tecnologías y al alumnado se le 
solicita mayor concentración y voluntad para el aprendizaje autónomo (Cabero, 2009). En este sentido, Jones \& Shao (2011) insisten en la necesidad de adaptación del profesorado a la nueva generación de estudiantes, nativos digitales, con la finalidad de que sus planteamientos metodológicos no queden obsoletos.

La adaptación de la educación al e-learning proporciona un acceso más flexible a los materiales educativos y permite el autoaprendizaje, convirtiendo al estudiante en el protagonista de la construcción de sus conocimientos, ventaja que destacan Viloria y Hamburger (2019) en la educación superior. La educación a distancia a través de las plataformas virtuales ha sido reconocida en la última década, tal y como apuntan Moreira et al. (2017), "como un método adecuado y un buen recurso para abordar los problemas a los que se enfrenta el mundo globalizado en materia de formación continua y desarrollo de habilidades tecnológicas y sociales» (p. 40).

En una revisión bibliográfica de publicaciones científicas en materia de educación a distancia, Gutiérrez y Borja (2016) concluyen que el e-learning se ha consolidado como una línea de investigación importante en la que hay que seguir avanzando, apelando a los «vacíos» existentes en la literatura especializada. La publicación de investigaciones en esta materia emerge a principios del año dos mil, concentrándose buena parte de las mismas en la primera década del siglo.

\subsection{El rol del docente, el rol del estudiante y la comunicación en la educación virtual}

Tal y como señala Aliste (2007), los avances en la tecnología vienen a entregarnos las herramientas necesarias para poder desarrollar una educación conveniente fuera del espacio presencial. No obstante, los nuevos entornos virtuales «(...) nos conducen irremediablemente a una transformación crítica de los aspectos fundacionales de las estructuras de la Comunicación Educativa o Educomunicación» (p. 7). Considerando la comunicación como la base fundamental de cualquier práctica educativa, se hacen necesarios estudios que profundicen en esta materia y que aporten conocimientos para su mejora.

En los nuevos espacios educativos es determinante planificar las situaciones de interacción que puedan darse con el alumnado con el fin de conseguir impactos cognitivos y sociales adecuados. En este sentido, Vilanova (2016) identifica cuatro tipos de interacciones posibles cuya correcta gestión garantizarían una educación efectiva, éstas son: alumno-instructor, que es la que nos ocupa en esta investigación; alumno-contenido; alumno-alumno y alumno-interfaz (p. 78).

La Teoría Transaccional de Moore $(1991 ; 1993)$ destaca el importante papel de las interacciones en los procesos de comunicación en la educación a distancia, siendo una de las aportaciones más importante, por parte del autor, el entender como causa del desfase en los procesos de enseñanza-aprendizaje la distancia de comunicación por encima de la distancia física entre profesor y alumno.

La interacción aparece asociada a la motivación (Mahle, 2011), a la persistencia y a la satisfacción del estudiante (Joo et al., 2011). La motivación se presenta como uno de los grandes desafíos en la educación a distancia. Usoro \& Abid (2008) señalan la necesidad de proporcionar una experiencia de aprendizaje altamente estimulante para evitar la deserción del alumno. En esta misma línea, Núñez-Urbina (2020) identifica la motivación, junto a los problemas de comunicación o la falta de integración social y académica, como algunas de las principales 
razones del abandono de los estudiantes en la modalidad de enseñanza virtual. El profesor deja de ser un mero transmisor de conocimientos para trasladar la responsabilidad del aprendizaje al estudiante (Vilanova, 2016) y convertirse en su orientador. Como señalan Galvis \& Pedraza (2016), la relación profesor y alumno pasa de ser vertical y unidireccional a un modelo de intercambio más horizontal. El docente, en su nuevo rol, debe apoyar el proceso de aprendizaje del alumno a través del uso de tareas de colaboración, la promoción de debates activos, el desarrollo del pensamiento crítico y de habilidades de investigación (Del Valle, 2019). Del Valle apunta, como uno de los retos a los que se enfrenta la educación en línea, a la necesidad de garantizar la plena convivencia y el desarrollo de habilidades emocionales, difíciles de trabajar por la falta del contacto directo. En este sentido, Vercher-Ferrándiz (2019) señala la gestión de la inteligencia emocional como competencia distintiva en la docencia telemática para la mejora del proceso de enseñanza-aprendizaje.

Otro de los aspectos relevantes en la función del docente como orientador es la tutoría virtual, campo, como apuntan Martínez et al. (2016), todavía poco desarrollado. Según los autores, existe una escasa utilización de las tutorías en el ámbito universitario, empleadas, principalmente, para resolver dudas del contenido de la materia en cuestión. Se trata de un uso reactivo del docente que responde a las demandas de consulta del estudiante. Por su parte, el alumnado valora de manera muy positiva la utilidad de la tutoría presencial frente a la virtual, pero, sin embargo, es la modalidad virtual la que más utilizan.

El uso de la tutoría virtual debe ir más allá de la resolución de dudas. En la educación a distancia debe emplearse como una herramienta fundamental a la hora de crear y fortalecer el vínculo con el estudiante. En este sentido, Hernández-Sánchez et al. (2015) señalan la importancia de las tutorías afectivas a la hora de reforzar los estados emocionales del alumnado y aumentar el disfrute en el proceso de aprendizaje. Sagastume et al. (2018) destacan el uso preferente de la videoconferencia en el desarrollo de las tutorías virtuales considerando la oportunidad que brinda de un mayor acercamiento con el alumno.

En definitiva, debemos entender la educación a distancia como «un diálogo didáctico entre el profesor y el estudiante que, ubicado en un espacio diferente al de aquél, aprende de forma independiente y también colaborativa» (García, 2011, p. 257). En este diálogo cobra especial importancia el uso planificado de recursos que estimulen y fomenten la participación, así como la escritura, componente esencial de la comunicación (Sanz \& Zangara, 2012). Estudios como los de Viloria \& Hamburger (2019) destacan el uso mayoritario del correo electrónico como herramienta de comunicación o el papel fundamental de los foros de discusión para fomentar la reflexión y la interacción grupal (Lapadat, 2002; Fariza, 2010).

En cuanto a la percepción que tienen los estudiantes del uso de las TIC en su proceso de formación, García-Valcárcel \& Tejedor (2017) concluyen con la valoración positiva que realiza el alumnado respecto a la incidencia de las TIC en el desarrollo de sus estrategias de aprendizaje, facilitando las tareas académicas. Igualmente, destacan que son los estudiantes con mejores calificaciones los que ofrecen una mejor valoración.

Los nuevos escenarios de enseñanza virtual suponen una oportunidad para una enseñanza accesible, para el aprendizaje autónomo, para el trabajo colaborativo y para el desarrollo de las habilidades sociales y tecnológicas que exigen los nuevos tiempos. No obstante, demandan un fuerte compromiso de aquellos que participan de esta formación. El alumnado se convierte en 
actor principal de su proceso de aprendizaje, situando al docente en el papel de guía en su formación.

\section{RESULTADOS}

Los resultados se estructuraron de la siguiente forma: por un lado, los datos arrojados a partir de la encuesta realizada al profesorado y, por otro, la información obtenida de la aplicación del cuestionario al alumnado que ha estado inmerso en el proceso de enseñanza telemática durante la COVID-19.

\subsection{Percepción de la enseñanza telemática por el profesorado universitario}

El cuestionario fue contestado por un $54 \%$ de profesoras y un $46 \%$ de profesores, siendo en su mayoría pertenecientes a universidades de titularidad pública, un $82 \%$, frente al $18 \%$ de universidad privada. Respecto a la edad del profesorado encuestado, la gran mayoría pertenece al rango comprendido entre los 35 y 45 años y los 45 y 55 años, sumando ambos el $67 \%$. Por su parte, la rama de conocimiento mayoritaria a la que se circunscribe el profesorado encuestado corresponde a Ciencias Sociales y Jurídicas, con un 74\%, seguida de Artes y Humanidades (11\%), Ciencias (7\%), Ingeniería y Arquitectura (5\%) y, en menor medida, la rama de Ciencias de la Salud (3\%). Respecto a las Comunidades Autónomas, los docentes andaluces y madrileños han sido los más participativos en el estudio con un $67 \%$ y un $12 \%$ respectivamente.

La mayoría de los encuestados, un 56\%, han manifestado conocer, aunque no en su totalidad, las plataformas y recursos puestos a su disposición por su centro o universidad durante la pandemia. Solo el $27 \%$ han afirmado conocerlas en su totalidad, mientras un $17 \%$ señalaban no saber de su existencia.

Por su parte, solo el $14 \%$ del profesorado ya hacía uso como medio habitual, antes de la COVID19, de las plataformas y recursos digitales que los centros o universidades ponen a disposición de la enseñanza telemática, en contraposición al $23 \%$ de los docentes que no las utilizaban con anterioridad a la pandemia. La mayor parte del profesorado universitario, un 63\%, hacía uso solo de algunas plataformas y recursos digitales (no de todos), por lo que la situación de excepcionalidad sobrevenida les ayudó a conocer otros medios para desarrollar la docencia.

Respecto al nivel de alfabetización digital antes del confinamiento, el $61 \%$ de profesorado considera que su nivel era óptimo (siendo "bueno" para el $44 \%$ de los encuestados y "muy bueno" para el 17\%). Un $29 \%$ estiman que su nivel era "regular" y solo el $10 \%$ del profesorado afirman contar con un nivel de alfabetización "malo" antes de la pandemia.

Las herramientas más utilizadas por el profesorado para comunicarse con su alumnado durante la COVID-19, para la explicación y el debate de los contenidos, así como para la resolución de las dudas relacionadas con el temario, han sido el email (99\%) y la videoconferencia (96\%), siendo estas dos las que consideran que han funcionado mejor en el proceso de enseñanza-aprendizaje. Por su parte, las herramientas menos empleadas por el profesorado y peor valoradas en cuanto a su efectividad han sido los vídeos de YouTube de creación propia, los blogs, las wikis y las redes sociales. (Véase los gráficos 1 y 2). 


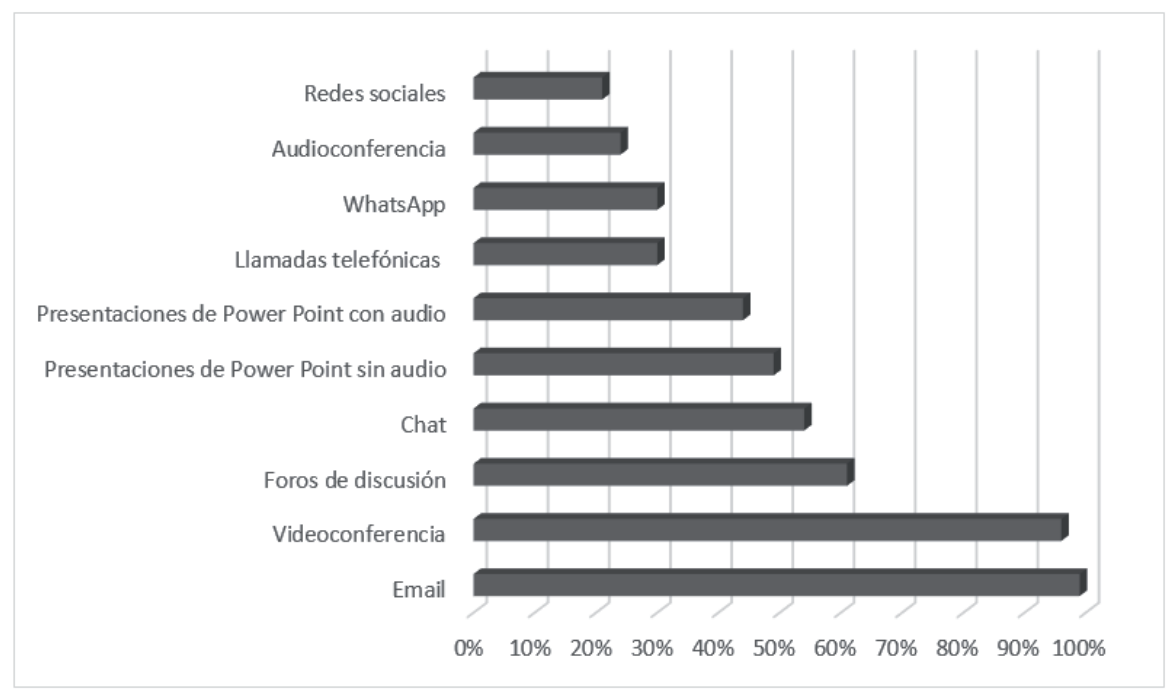

Figura 1. Porcentaje de herramientas más utilizadas por el profesorado para comunicarse con el alumnado

Fuente: elaboración propia.

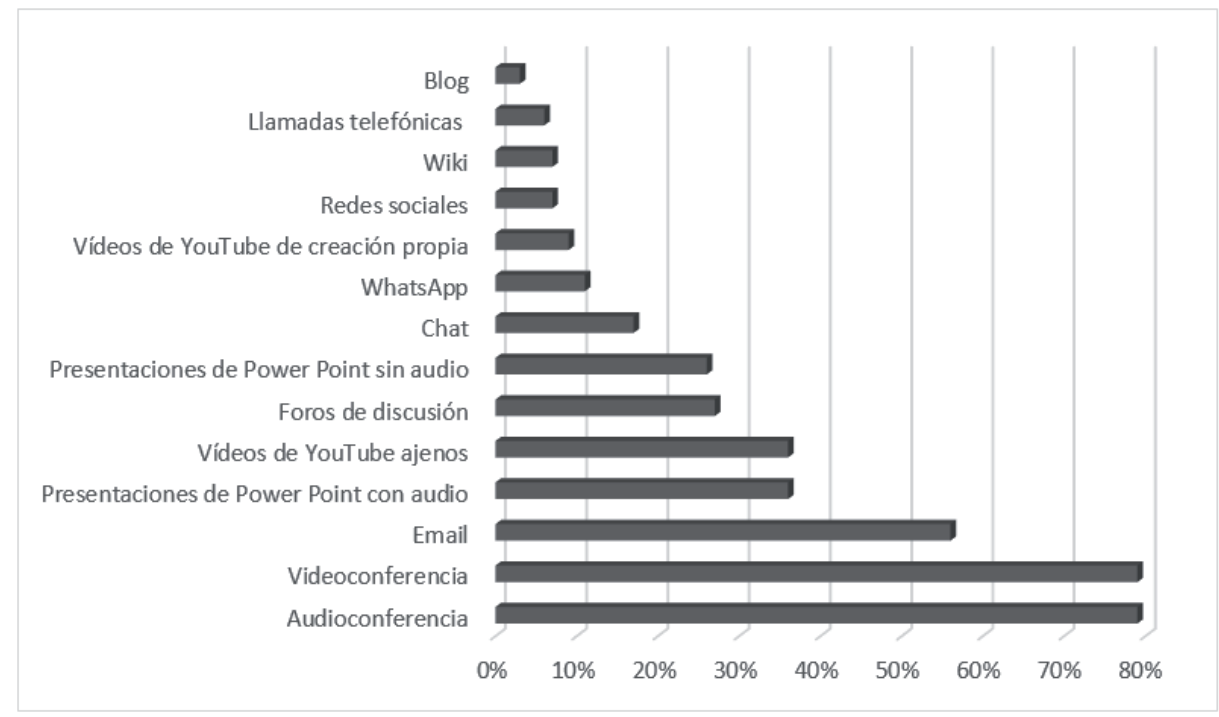

Figura 2. Porcentaje de herramientas mejor valoradas por el profesorado en cuanto a su efectividad Fuente: elaboración propia.

En relación a las herramientas y/o recursos que han funcionado mejor en la comunicación social, la desarrollada entre el docente y el alumnado para el fomento de las relaciones personales y el vínculo afectivo, el correo electrónico y la videoconferencia han sido también los más efectivos, con un $70 \%$ y un $66 \%$ respectivamente. Otros recursos han sido también mencionados por el profesorado, si bien, en porcentaje menor. Así, chats y foros de discusión, son tenidos en consideración por el $16 \%$ de docentes, como herramientas que funcionan a la hora de fomentar la relación docente-discente. Un 14\% apunta a WhatsApp y un 13\% a llamadas telefónicas. Las redes sociales son mencionadas por un $11 \%$ de profesores y la audioconferencia por un $10 \%$.

Con respecto a los inconvenientes encontrados en la enseñanza telemática a la hora de comunicarse con sus estudiantes respecto a la enseñanza presencial, sobresale la desventaja que supone la falta de estímulo del alumnado al no encontrarse en el aula (63\%), la consecuente 
dificultad a la hora de implicar al estudiante (57\%) y de conectar emocionalmente con él (56\%), así como los problemas derivados de la conexión a la red (59\%). En cuanto a las ventajas que perciben en la modalidad a distancia, es reseñable el hecho de que el $47 \%$ de los docentes ( 99 de los 210) no ha encontrado ventaja alguna en la virtualidad respecto a la presencialidad. En este sentido, las ventajas más destacables son la implicación mayor que ha supuesto en el estudiantado, así como la mayor participación del mismo durante las clases y debates. (Véase los gráficos 3 y 4 ).

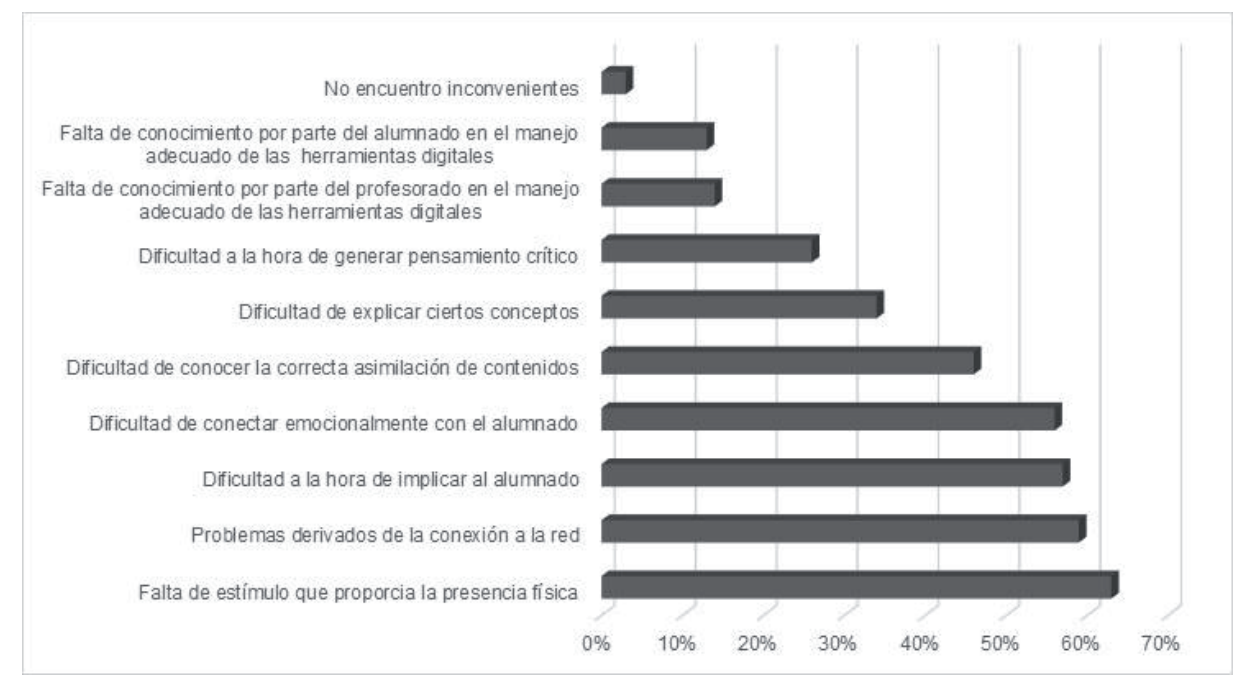

Gráfico 3. Inconvenientes principales de la enseñanza telemática a la hora de comunicarse con el alumnado

Fuente: elaboración propia.

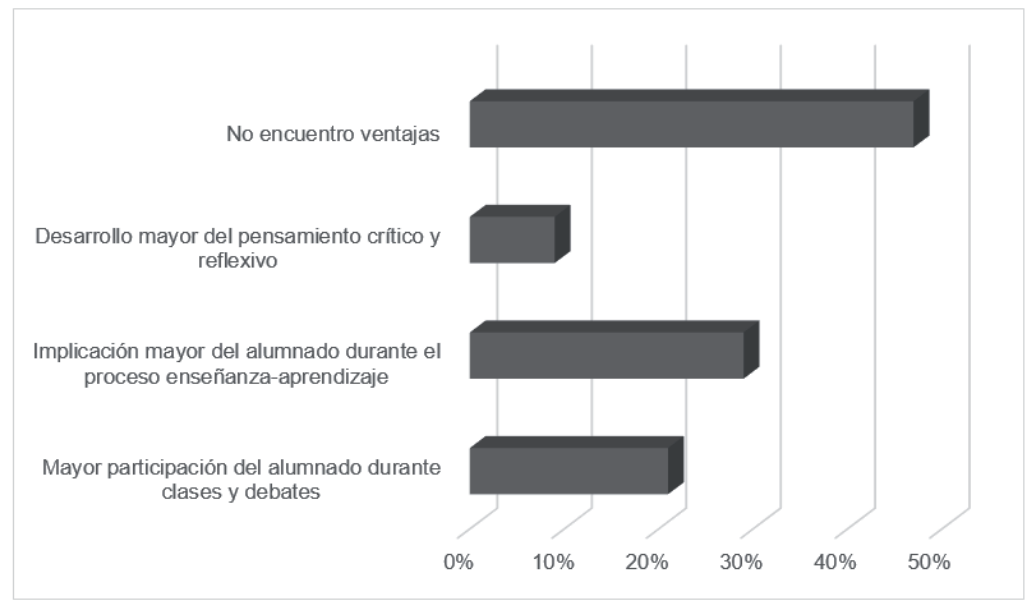

Gráfico 4. Ventajas principales de la enseñanza telemática a la hora de comunicarse con el alumnado Fuente: Elaboración propia

En cuanto a la valoración que el docente realiza de la comunicación desarrollada con el alumnado durante la enseñanza telemática provocada por el estado de alarma, más de la mitad del profesorado realiza una valoración positiva, catalogándola un $41 \%$ de los encuestados de "buena" y un $10 \%$ de "muy buena". No obstante, consideran que la enseñanza a distancia 
empeora tanto la comunicación didáctica como la comunicación social profesor-alumno frente a la educación presencial.

Respecto al grado de dificultad encontrado para llevar a cabo la enseñanza online, destaca una dificultad media alta. De esta forma, el $27 \%$ han encontrado una dificultad media, un $30 \%$ manifiestan un grado alto de dificultad y el $9 \%$ muy alto. Solo un $16 \%$ de los docentes encuestados manifiestan no haber tenido ninguna dificultad.

\subsection{Percepción de la enseñanza telemática por el alumnado universitario}

El alumnado participante ha estado en su mayoría conformado por mujeres (73\%) y la franja de edad mayoritaria se comprende entre los 18 y los 25 años (89\%). El 93\% del alumnado encuestado está cursando Grado y el $7 \%$ restante estudios de Posgrado. Respecto a la titularidad de las universidades a las que pertenecen los 501 estudiantes participantes en el cuestionario, la gran mayoría (94\%) pertenece a universidades públicas, mientras que el $6 \%$ estudia en universidades privadas. A su vez, los estudiantes pertenecen en su mayor parte a la rama de Ciencias Sociales y Jurídicas (67\%), seguidos del $22 \%$ correspondiente a Artes y Humanidades. El $5 \%$ estudia Ciencias, el $4 \%$ lo hace en Ingeniería y Arquitectura y solo un $2 \%$ cursa Ciencias de la Salud. En cuanto a la procedencia, los estudiantes andaluces han sido los que más han participado en el estudio (74\%), seguidos de los estudiantes gallegos (11\%).

Por regla general, el alumnado considera que su nivel de alfabetización digital antes del confinamiento era "bueno" (47\%) y "muy bueno" (19\%). Por tanto, un 66\% del alumnado encuestado apuntan a un buen nivel.

El $60 \%$ del estudiantado apunta a que el profesorado ya utilizaba de manera frecuente las plataformas virtuales y los recursos digitales disponibles para el desarrollo de sus clases frente a un $40 \%$ que apuntan a que no hacían uso del mismo.

El email ha sido, con un $86 \%$ de respuestas, la herramienta más utilizada por el profesorado para comunicarse con su alumnado a la hora de aclarar dudas del contenido de las materias y establecer feedback. Le siguen, por encima de la media, la videoconferencia (81\%), las presentaciones de Power Point $u$ otras aplicaciones con audio explicativo (67\%) y los foros de discusión (57\%). Por su parte, las herramientas que mejor han funcionado durante el proceso de enseñanza-aprendizaje, desde el punto de vista del alumnado, han sido la videoconferencia, con un $74 \%$ de las respuestas, y el correo electrónico (33\%). Las herramientas y recursos menos valorados por el estudiantado en la comunicación con el profesorado han sido las redes sociales (5\%), los vídeos de YouTube ajenos, las Wikis, las llamadas telefónicas y el WhatsApp (4\% cada una). La herramienta peor valorada ha sido el blog (1\%) (véase los gráficos 5 y 6). 


\section{Gráfico}

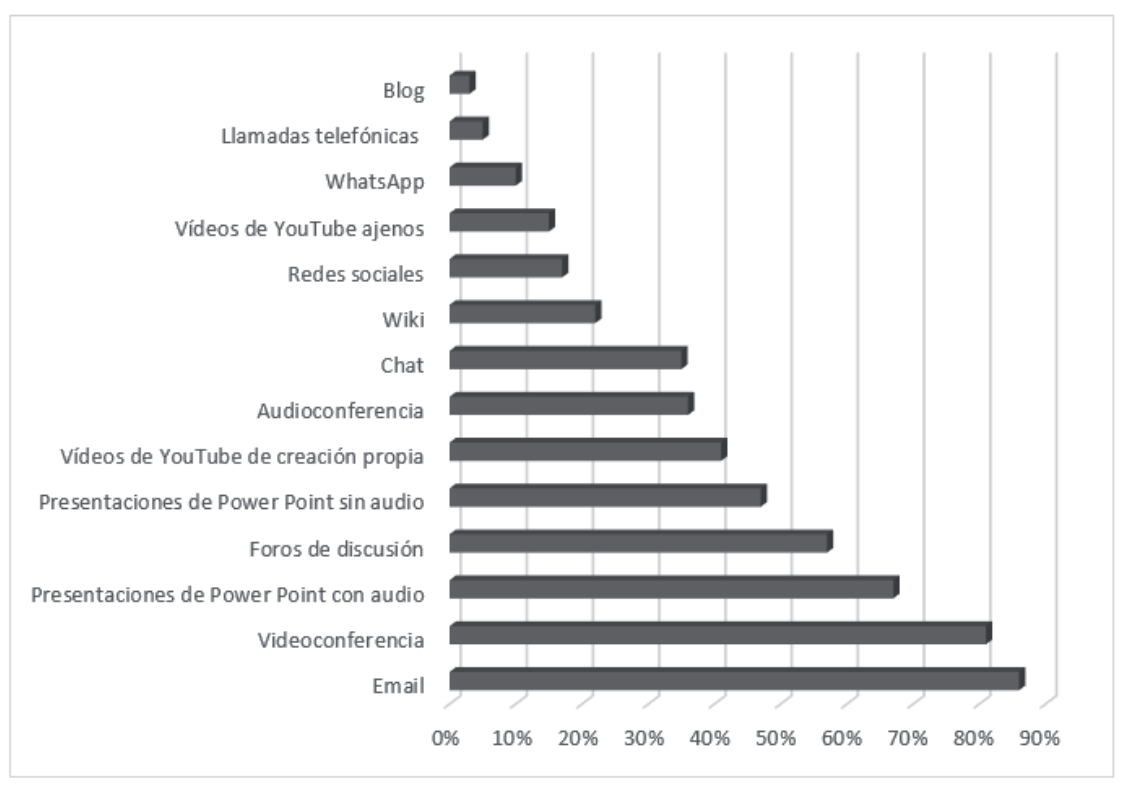

Herramientas más utilizadas para dar Feedback y explicar contenidos

Fuente: elaboración propia.

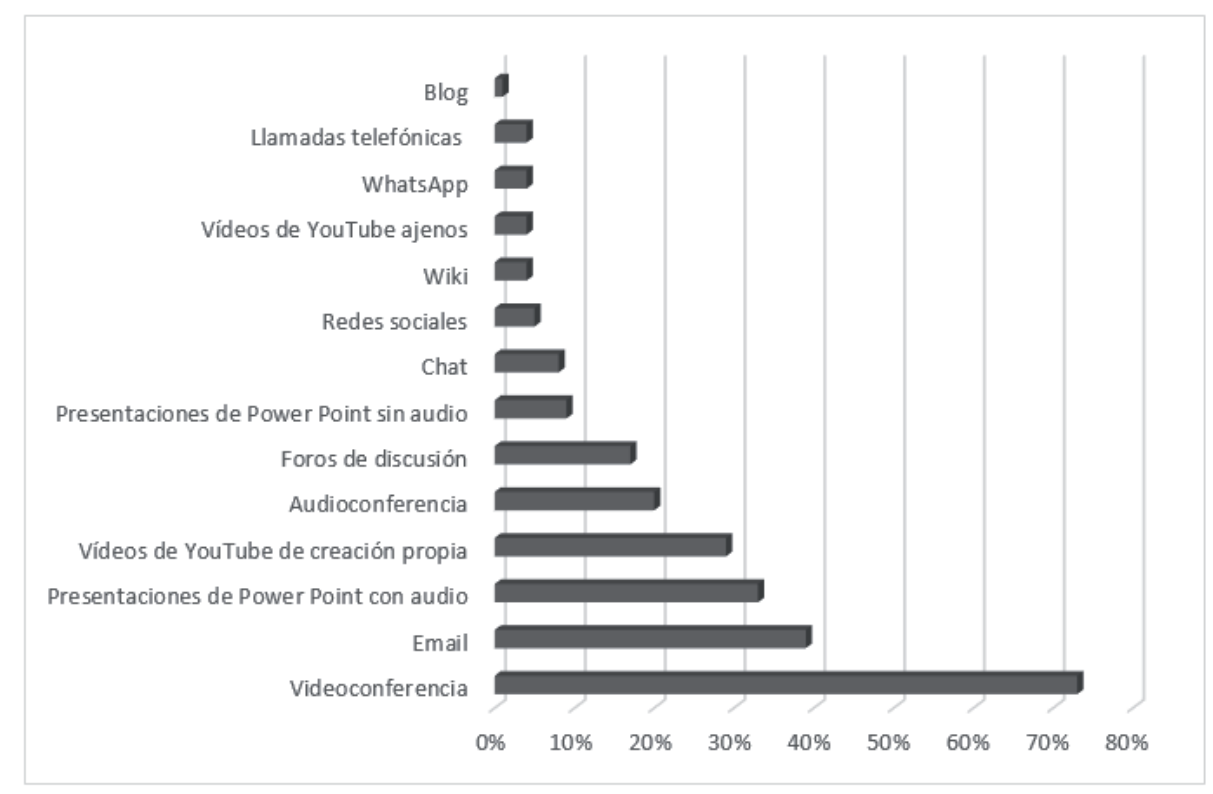

Gráfico 6. Valoración de las herramientas que mejor han funcionado en la comunicación social según el alumnado

Fuente: elaboración propia.

Las herramientas que el alumnado considera que han funcionado mejor en la comunicación social desarrollada con el profesorado son, en primer lugar, el correo electrónico, con un 68\%, seguido de la videoconferencia, con un $47 \%$ de las respuestas. Las herramientas que el estudiantado cree que menos han funcionado en la comunicación social mantenida con sus docentes son las wikis, los blogs, las redes sociales y las llamadas telefónicas (véase el gráfico 7). 


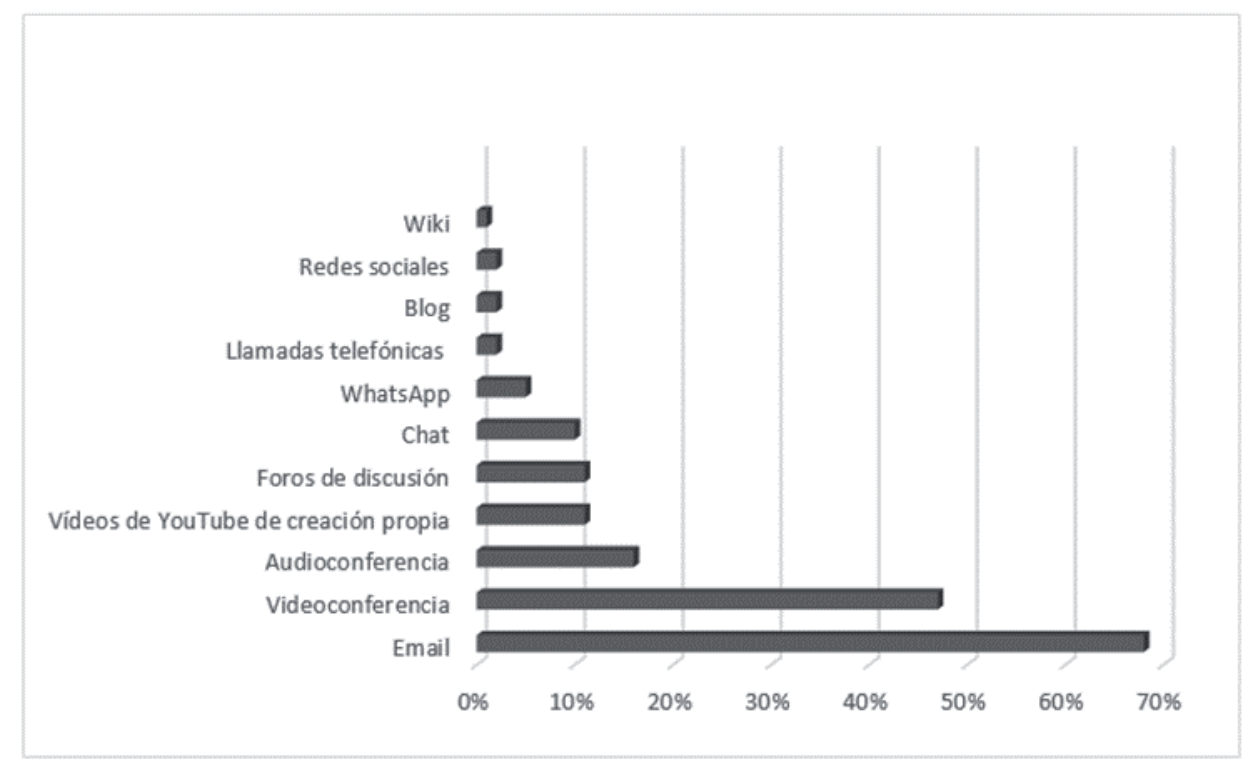

Gráfico 7. Valoración de las herramientas que mejor han funcionado en el proceso enseñanzaaprendizaje

Fuente: elaboración propia.

Han sido diversos los inconvenientes que el estudiantado ha encontrado en la enseñanza telemática en la comunicación desarrollada durante las explicaciones de contenido, tutorías o las interacciones en general que mantienen con el profesorado, respecto a la enseñanza virtual. El principal inconveniente señalado, por un $65 \%$ del alumnado participante, se encuentra en la dificultad de entender y asimilar ciertos conceptos muy técnicos sin el apoyo de prácticas presenciales. Le siguen los problemas de conexión a la red (61\%). El tercer inconveniente que han remarcado los estudiantes radica en la falta de presencia física de sus compañeros y compañeras (45\%). El $32 \%$ de alumnado señala el pudor que le genera participar en una videoconferencia con cámara y/o audio. El 30\% de los estudiantes también apunta como inconveniente la falta de un espacio físico adecuado para poder recibir las clases de forma cómoda. Un $22 \%$ señala las limitaciones con las que cuentan a nivel particular por no contar con dispositivos adecuados (ordenadores, conexión a Internet...). Un 10\% reconocen como inconveniente su falta de conocimiento en el manejo adecuado de las herramientas digitales existentes. Hay otros inconvenientes destacables en los apartados "Otros" como es el acceso a ciertos materiales que en carreras relacionadas con Artes o Ciencias son necesarios; también destacan la excesiva carga de trabajo sobrevenida con la enseñanza virtual, la falta de concentración, el escaso interés de algún docente en concreto y la ausencia de explicaciones.

En relación a las ventajas que el alumnado ha encontrado en la enseñanza telemática en la comunicación que se desarrolla durante las explicaciones del contenido, tutorías y/o interacciones con el profesorado respecto a la presencialidad, cabe destacar que el $52 \%$ de encuestados no ve ventaja alguna. El $32 \%$ considera que gracias a la virtualidad, el profesorado ha intensificado el feedback con el alumnado. Por su parte, el $26 \%$ de discentes apuntan a que su comunicación con el profesorado se ha vuelto más habitual con la virtualidad y el $14 \%$ manifiestan que su participación en las clases y en los debates ha aumentado respecto a la presencialidad. Un $3 \%$ de estudiantes añaden otras ventajas como el hecho de que hay clases que se guardan y pueden visionarse tantas veces como se desee; a su vez, apuntan a que los 
horarios de las interacciones entre alumnado y profesorado a veces se pactan para mayor comodidad de las partes.

La experiencia del alumnado con la enseñanza telemática respecto a la comunicación mantenida con el profesorado no es del todo grata, tal y como manifiesta el 35\% del estudiantado encuestado (siendo "muy mala" para el 6\% y "mala" para el $29 \%$ ). A ello hay que añadir el catalogar dicha comunicación con sus profesores como "regular" por un 37\% de los encuestados. Solo un $\mathbf{2 8} \%$ de estudiantes consideran óptima la comunicación mantenida. Así, el $25 \%$ considera que ha sido "buena" y solo un $3 \%$ la define como "muy buena". En definitiva, los estudiantes consideran, en su mayoría, que la enseñanza a distancia empeora tanto la comunicación didáctica como la comunicación social profesor-alumno frente a la educación presencial.

Respecto al grado de dificultad encontrado para llevar a cabo la enseñanza online, destaca una dificultad media alta. De esta forma, el $27 \%$ han encontrado una dificultad media, un $30 \%$ manifiestan un grado alto de dificultad y el $9 \%$ muy alto. Solo un $16 \%$ de los estudiantes encuestados manifiestan no haber tenido ninguna dificultad.

\section{DISCUSIÓN Y CONCLUSIONES}

En consonancia con estudios precedentes, esta investigación acredita (Echeverría, 2002; Lorenzo et al., 2019) que las nuevas tecnologías nos proporcionan los recursos y herramientas necesarias para la concepción de nuevos espacios sociales de aprendizaje. Las transformaciones que ha sufrido la sociedad suponen que sea necesario un cambio significativo en la concepción del proceso de enseñanza-aprendizaje y de las funciones a cubrir por las distintas instituciones educativas (Chamizo \& Fernández, 2013).

2020 ha sido un año en el que las sociedades han tenido que asistir a una educación online de emergencia como consecuencia de la crisis sanitaria COVID-19. Nos hallamos pues, como señalan Hodges et al. (2020), ante un ERT o Emergency Remote Teaching, una enseñanza remota de emergencia (ERE), una alternativa de dar clase debido a circunstancias de crisis que contrasta con experiencias educativas planteadas desde un principio y diseñadas para ser online. La educación sobrevenida por la pandemia ha llevado aparejadas, como también señalan los autores anteriores, soluciones de enseñanzas remotas aplicadas a metodologías elaboradas para clases presenciales o, en algunos casos, híbridas, que volverían a su status quo inicial una vez finalizada la crisis. Este hecho concreto, de adaptación precipitada de la enseñanza presencial a la enseñanza en línea, no ha hecho más que alimentar la idea de la debilidad de la enseñanza telemática frente a la presencial.

La dificultad encontrada por la mayoría de docentes y alumnado, a la hora de llevar a cabo la enseñanza online, ha sido media y alta, lo que contrasta con el nivel de alfabetización digital que dicen poseer, catalogado por la mayor parte de encuestados como óptimo. En este sentido, entendemos que la dificultad manifestada no viene asociada a la capacitación en el manejo de recursos virtuales sino, más bien, al estado de improvisación al que se ha visto abocada la adaptación de la enseñanza presencial a la virtual.

La comunicación entre profesores y estudiantes en la enseñanza telemática se ha llevado a cabo mediante recursos y/o plataformas con los que las universidades ya contaban antes del confinamiento, si bien solo eran conocidas en su totalidad y usadas de manera habitual por un 
número reducido de docentes universitarios, lo que significa que gran parte de ellos hacían uso solo de algunos recursos y/o plataformas como apoyo puntual de sus clases presenciales. La pandemia, por tanto, ha traído consigo que los profesionales hayan tenido que reciclarse y explorar herramientas que, aunque previamente estaban a su disposición, no utilizaban en su totalidad para su práctica docente. Por otro lado, hay que considerar que la manera apresurada de adaptación al nuevo escenario de aprendizaje en línea no ha permitido el conocimiento y la planificación de los recursos telemáticos necesarios para una enseñanza efectiva. El nuevo escenario deja en evidencia, así, la necesidad de una adaptación mayor, en conocimiento y uso, por parte del profesorado a los recursos que ofrecen las nuevas tecnologías.

Las herramientas de comunicación más utilizadas entre docente-discente han sido el correo electrónico, tal y como también apuntan las conclusiones de Viloria \& Hamburger (2019), y la videoconferencia, que también han sido catalogadas como aquellas que mejor han funcionado en el proceso de enseñanza-aprendizaje, así como en el fomento de las relaciones personales y el establecimiento de vínculos entre ambos agentes del proceso educativo. En definitiva, se trata de herramientas que, como indican Sagastume et al. (2018) al referirse a las videoconferencias, permiten una comunicación más cercana, una comunicación de contacto entre profesor y alumno, proporcionando el feedback tan necesario en la educación a distancia.

Entre los principales inconvenientes señalados por el personal docente se encuentra la falta de motivación por parte del alumnado, la dificultad a la hora de implicarlo y la falta de conexión emocional con el mismo, aspectos estos relevantes para el desarrollo de un adecuado proceso de enseñanza-aprendizaje. Por su parte, el estudiantado también encuentra inconvenientes en este tipo de enseñanza respecto a la presencial, destacando la dificultad de asimilar y entender determinados términos, así como la mala conexión a Internet y la ausencia de compañeros en su proceso de aprendizaje. El pudor que puede suponer ponerse ante una cámara y/o un audio así como la falta de un espacio adecuado para poder recibir las clases de forma óptima también son aspectos señalados por los estudiantes.

La mitad, aproximadamente, del profesorado y del alumnado encuestado no ha encontrado ventaja alguna en la educación a distancia desarrollada durante el estado de alarma por las universidades españolas. Los docentes que apuntan ventajas manifiestan una mayor implicación del alumnado, así como el incremento de la participación en las clases virtuales respecto a las presenciales. En la misma línea, el alumnado considera que la enseñanza telemática ha provocado la intensificación del feedback del profesorado con sus estudiantes, lo que lleva aparejado un aumento de la comunicación entre ambos. Otra de las ventajas que apuntan los alumnos es el incremento de su participación en las clases virtuales gracias al fomento del debate por parte del profesorado.

En definitiva, y dando respuesta a las preguntas de investigación planteadas, podemos concluir que:

(P1) La valoración global de la comunicación llevada a cabo entre el profesorado y el alumnado diverge según se trate de un rol u otro. Así, existe discrepancia entre docentes y estudiantes respecto a la comunicación mantenida ya que la gran mayoría de los profesores valora de forma positiva la comunicación con su alumnado en la virtualidad impuesta por la pandemia. Por el contrario, destaca la valoración negativa de la misma por parte de una gran mayoría del estudiantado encuestado. En lo que sí coinciden ambos agentes del proceso 
educativo es que el vínculo y las relaciones personales empeoran con la virtualidad, así como la comunicación llevada a cabo durante las explicaciones de los contenidos, sobre todo, al tratarse de conceptos técnicos.

(P2) Profesores y estudiantes han afirmado, en su mayoría, haberse encontrado con un nivel medio-alto de dificultad a la hora de enfrentarse a la enseñanza telemática. En este sentido, debemos considerar el nivel medio bajo en el conocimiento y uso de los recursos telemáticos disponibles en los Centros por parte del profesorado en los momentos previos a la pandemia. La dificultad podría deberse al improvisado escenario en el que han actuado. Docentes y estudiantes se han visto abocados a una enseñanza a distancia para la que no estaban preparados, con unas materias cuyos programas estaban diseñados para una educación presencial y no exclusivamente virtual.

(P3) Las herramientas de comunicación más utilizadas y mejor valoradas en el proceso de enseñanza-aprendizaje por parte de ambos actores han sido la videoconferencia y el correo electrónico.

(P4) Alumnos y profesores encuentran más inconvenientes que ventajas en la enseñanza a distancia desarrollada durante el estado de alarma.

El estudio abarcado nos ha permitido conocer y valorar la adaptación de las universidades españolas de carácter presencial a la modalidad de educación a distancia, desde la óptica de la comunicación desarrollada entre docentes y estudiantado. Unos resultados que nos muestran una radiografía bastante precisa del nivel de comunicación desarrollada en el entorno digital por parte de un importante volumen de centros universitarios; si bien es cierto que hay que destacar el predominio de participantes de la comunidad andaluza en la muestra resultante.

De manera prospectiva, pueden considerarse futuras líneas de investigación aquellas llamadas a estudiar acciones que permitan tanto a instituciones educativas como a gobiernos la mejora de la educación telemática y, por ende, la comunicación entre el docente y el estudiantado en los nuevos marcos virtuales. La comunicación, tal y como hemos apuntado en esta investigación, es un elemento clave para el desarrollo de un proceso de enseñanza-aprendizaje eficaz. Con esta investigación se han identificado las herramientas más o menos empleadas, más o menos eficaces y más o menos valoradas en el proceso de comunicación entre docentes y alumnado. Necesitamos nuevas investigaciones que permitan profundizar en cada una de estas herramientas para conocer el mejor uso que pueda hacerse de las mismas de cara al desarrollo no sólo de una comunicación didáctica, sino también, y de manera muy especial, de una comunicación social que permita conectar emocionalmente a los agentes del proceso. Un aspecto éste relevante en un escenario educativo donde lo digital deteriora las relaciones personales.

\section{Referencias}

Aliste, C. (2007). Modelo de comunicación para la enseñanza a distancia. Análisis experimental de una plataforma de e-learning [Tesis doctoral, Universidad Autónoma de Barcelona]. https://bit.ly/3qMrc5M

Cabero, J. (2009). Los nuevos escenarios y las nuevas modalidades de formación: Las aportaciones desde las nuevas y antiguas tecnologías. En J. Tejada y otros (Coord.), IV Congreso de formación para el trabajo (pp. 187-207). Tornapunta Ediciones.

Chamizo, R. y Fernández, M.J. (2013). Del aula al espacio virtual: Nuevas fórmulas para el aprendizaje 
cooperativo. En J. Díaz-Cuesta (Coord.), Estrategias innovadoras para la docencia dialógica y virtual (pp.257-279). Visión Libros.

Del Valle, M.A. (2019). El manejo de la inteligencia emocional en las aulas virtuales y su impacto socio educacional: el camino de entornos urbanos inteligentes hacia entornos urbanos emocionales. En Actas Icono14: VII Congreso Internacional Ciudades Creativas (pp. 502-517). Asociación de Comunicación y Nuevas Tecnologías.

Durán, R.A. (2016). La Educación Virtual Universitaria como medio para mejorar las competencias genéricas y los aprendizajes a través de buenas prácticas docentes [Tesis doctoral, Universidad Politécnica de Cataluña]. https://bit.ly/3tmBf2W

Echeverría, B. (2002). Gestión de la Competencia de Acción Profesional. Universidad de Barcelona.

Esquivel, A.S. y Canto, P.J. (2018). Tipos de interacciones en un ambiente virtual de aprendiza-je entre estudiantes, profesores y contenidos. Revista Valera, 18(51), 263-277. https://bit.ly/38EqEbV

Fariza, N., Razak, N.A. \& Aziz, J. (2010). E-learning: analysis of online discussion forums in promoting knowledge construction through collaborative learning. Wseas Transactions on Communications, 9(1), 53-62. https://bit.ly/30FT9Bt

Forsyth, H., Pizzica, J., Laxton, R., \& Mahony, M. J. (2010). Distance education in an era of elearning: Challenges and opportunities for a campus-focused institution. Higher Education Research and Development, 29(1), 15-28. https://doi:10.1080/07294360903421350

Galvis, A. H., y Pedraza, L. d. (2013). Desafíos del e-learning y del blearning en educación superior. En N. Arboleda Toro y T. Rama Vitale (Ed.), La educación superior a distancia y virtual en Colombia: nuevas realidades (113-154). ACESAD.

García, L. (2011). Perspectivas teóricas de la educación a distancia y virtual. Revista Española de Pedagogía, 249, 255-271. https://bit.ly/3qI2J1j

García-Valcárcel, A. y Tejedor, F. J. (2017). Percepción de los estudiantes sobre el valor de las TIC en sus estrategias de aprendizaje y su relación con el rendimiento. Educación XX1, 20(2), 137-159, https://doi:0.5944/educXX1.19035

Gutiérrez, G.F. y Borja, Y.A. (2016). Elearning. Una visión de las publicaciones en revistas de alto impacto. Revista Publicando, 3(8), 162-169. https://bit.ly/30HWikn

Hernández-Sánchez, Alba, M., y Ortega, J. A. (2015). Aprendizaje Electrónico Afectivo: un modelo Innovador para Desarrollar una Acción Tutorial Virtual de Naturaleza Inclusiva. Formación Universitaria, 8(2), 19-26, https//doi:10.4067/S0718-50062015000200004

Hodges, Ch., Moore, S., Lockee, B., Trust, T. \& Bond, A. (2020). The Difference Between Emergency Remote Teaching and Online Learning. Educause Review. https://bit.ly/30F90Am

Jones, Ch. \& Shao, B. (2011). The net generation and digital natives: implications for higher education. Higher Education Academy. https://bit.ly/3bNSsg5

Joo, Y. J., Lim, K. Y. \& Kim, E. K. (2011). On line university students' satisfaction and persistence: Examining perceived level of presence, usefulness and ease of use as predictors in a structural model. Computers \& Education, 57(2), 1654-1664.

http://dx.doi.org/10.1016/j.compedu.2011.02.008.

Lapadat, J. C. (2002). Written Interaction: A Key Component in Online Learning. Journal of ComputerMediated Communication, 7(4), https://doi.org/10.1111/j.1083-6101.2002.tb00158.x

Lorenzo, M.M., Ferraces, M.J., Pérez, C. y Naval, C. (2019). El profesorado universitario ante el aprendizaje-servicio: variables explicativas. Revista de Educación, 386, 37-61, https://doi:10.4438/1988-592X-RE-2019-386-426

Mahle, M. (2011). Effects of interaction on student achievement and motivation in distance education. Quarterly Review of Distance Education, 12(3), 207-215. https://bit.ly/2OvZsFp

Martínez, P., Pérez, J. y Martínez, M. (2016). Las TICS y el entorno virtual para la tutoría universitaria. Educación XX1, 19(1), 287-310, https://doi:10.5944/educXX1.13942 
Moore, M. G. (1991). Editorial: Distance Education Theory. The American Journal of Distance Education, 5(3), 1-6.

Moore, M. G. (1993). Theory of transactional distance. En D. Keegan (Ed.), Theoretical principles of distance education, (22-29). Routledge.

Moreira, J.A., Reis-Monteiro, A. y Machado, A. (2017). La educación superior a distancia y el e-Learning en las prisiones en Portugal. Comunicar, 25(51), 39-49. https://doi.org/10.3916/C51-2017-04

Núñez, A. A. (2020). La educación en línea y el rol de la motivación. Revista Transdigital, 1(1). https://bit.ly/3bLvA0q

Palvia, Sh., Aeron, P., Gupta, P., Mahapatra, D., Parida, R., Rosner, R. \& Sindhi, S. (2018). Online Education: Worldwide Status, Challenges, Trends and Implications. Journal of Global Information Technology Management, 21(4), 233-241, https://doi:10.1080/1097198X.2018.1542262

Raya, E. y Gómez, M. (2018). Estudio comparativo sobre metodologías de enseñanza y aprendizaje en tres universidades británicas. En E. Domínguez Romero, J. Bobkina y M.L. Pertegal Felices (Coords), Alfabetización digital e informacional (pp. 409-426). Gedisa.

Sagastume, F., Morales, M., Amado, H. y Hernández, R. (2018). La importancia del tutor en los cursos virtuales: experiencia, buenas prácticas y recomendaciones. En Proceedings of the Digital World Learning Conference (pp. 91-97). Recuperado de https://bit.ly/30Fxf1v

Sanz, C. y Zangara, A. (2012). La escritura colaborativa como una e-actividad. En Actas del XVIII Congreso Argentino de la Ciencia de la Computación (580-588). Instituto de Investigación en Informática LIDI.

Usoro, A. \& Abid, A. (2008). Conceptualising Quality E-learning in Higher Education. E-learning, 5(1), 7588, http://dx.doi.org/10.2304/elea.2008.5.1.75

Vercher-Ferrándiz, M. L. (2019). La gestión de la inteligencia emocional como competencia distintiva en la docencia online para la mejora de la gestión del proceso enseñanza-aprendizaje en el ámbito de las ciencias sociales [Tesis doctoral. Universitat Politècnica de València]. https://bit.ly/3rNw3F8

Vilanova, G.E. (2016). Modelos de interacción en ambientes virtuales de aprendizaje en la Educación Superior. Sistemas, Cibernética e Informática, 13(1), 77-83. https://bit.ly/3rOnE4n

Viloria, H.A. y Hamburger, J. (2019). Uso de las herramientas comunicativas en los entornos virtuales de aprendizaje. Chasqui. Revista Latinoamericana de Comunicación, 140, 367-384. https://bit.ly/2OQCxVh

\section{Semblanza de los autores}

María Jesús Fernández Torres es Doctora en Ciencias de la Comunicación. Fue la doctora más joven de España. Departamento de Comunicación Audiovisual y Publicidad. Profesora de la Universidad de Málaga. Funcionaria en excedencia. Autora de cinco libros: Las Relaciones Públicas como gestión de la Comunicación en Movimientos Sociales. Análisis de la estrategia comunicativa de las ONG en España, Movimientos sociales y acción colectiva: pasado y presente, Protocolo y Relaciones Públicas, Protocolo de bolsillo: las reglas de oro y Atención al cliente, consumidor y usuario. Mantiene gran actividad investigadora y posee artículos en libros y revistas científicas. Revisora y evaluadora en revistas nacionales e internacionales, participante en grupos de investigación. Pertenece al Consejo de Redacción de la Revista Internacional de Relaciones Públicas.

Rocío Chamizo Sánchez es Profesora del departamento de Comunicación Audiovisual y Publicidad de la Universidad de Málaga, desde 2002. Docencia en el grado de Publicidad y Relaciones Públicas y en el Máster Interuniversitario en Dirección Estratégica e Innovación en Comunicación. Miembro del equipo editorial de la Revista Internacional de Relaciones Públicas. 
Sus líneas de investigación principales se centran en el desarrollo y mejora de las estrategias y técnicas de enseñanza-aprendizaje en la educación universitaria y en la comunicación turística y de ocio. En este sentido, destacamos la participación en proyectos tales como "Inteligencias Múltiples en la Enseñanza Universitaria en Comunicación"; "Metodologías y herramientas para la acción tutorial en grado y postgrado. Diseño y aplicación o "Aplicación de las TIC y el Campus Virtual para el aprendizaje cooperativo".

Rocío Sánchez Villarrubia es Profesora de Educación Secundaria en la especialidad de inglés y ha sido Profesora del departamento de Lenguas Modernas en el Mount St Mary's College en Sheffield, Inglaterra, durante cuatro años. Asesora lingüística en las etapas de infantil y primaria en la Comunidad de Madrid, en programa educativo en el que colaboran el Ministerio de Educación y el British Council. Sus líneas de investigación se centran en educación superior y educación no universitaria, en esta línea destacar la publicación "Relaciones Públicas y Programas De Movilidad en Educación Superior: Tendencias y Estrategias Comunicativas" y "Comunicación y alfabetización digital en educación durante la COVID-19". 